\title{
Molecular Docking Study of Aminoacyl-tRNA Synthetases with Ligand Molecules from Four Different Scaffolds ${ }^{\dagger}$
}

\author{
Nagakumar Bharatham, ${ }^{a}$ Kavitha Bharatham, ${ }^{b}$ Yuno Lee, Songmi Kim, Prettina Lazar, Ayoung Baek, Chanin Park, \\ Heesung Eum, ${ }^{\ddagger}$ Hyun-Joon Ha, ${ }^{\ddagger}$ Sae Young Yun, ${ }^{\S}$ Won Koo Lee, ${ }^{\S}$ Sunghoon Kim, ${ }^{\#}$ and Keun Woo Lee* \\ Division of Applied Life Science (BK21 Program), Environmental Biotechnology National Core Research Center \\ (EB-NCRC), Plant Molecular Biology and Biotechnology Research Center (PMBBRC), \\ Gyeongsang National University (GNU), Jinju 660-701, Korea. *E-mail: kwlee@gnu.ac.kr \\ ${ }^{\ddagger}$ Department of Chemistry and Protein Research Center for Bio-Industry, Hankuk University of Foreign Studies, \\ Yongin 449-791, Korea \\ ${ }^{\S}$ Department of Chemistry, Sogang University, Seoul 121-742, Korea \\ ${ }^{\#}$ Center for Medicinal Protein Network and Systems Biology, College of Pharmacy, Seoul National University, \\ Seoul 151-741, Korea \\ Received December 28, 2009, Accepted January 30, 2010
}

\begin{abstract}
Aminoacyl-tRNA synthetases (aaRSs) play vital roles in protein biosynthesis of living organisms and are interesting antibacterial drug targets. In order to find out new inhibitor candidate molecules as antibacterial agent, the binding modes of the candidate molecules were investigated at the active sites of aaRSs by molecular docking study. The docking simulations were performed with 48 compounds from four different scaffolds into the eight different aaRSs. The results show that scaffolds 3 and 4 compounds have consistently better binding capabilities, specifically for HisRS (E. coli) and IleRS (S. aureus). The binding modes of the best compounds with the proteins were well compatible with those of two ligands in crystal structures. Therefore, we expect that the final compounds we present may have reasonable aaRS inhibitory activity.
\end{abstract}

Key Words: aaRS (aminoacyl-tRNA synthetase), HisRS, IleRS, Antibacterial drug target, Molecular docking simulation

\section{Introduction}

Aminoacyl-tRNA synthetases (aaRSs) play vital roles in protein biosynthesis of all living organisms. When bacterial aaRSs are inhibited by antibacterial drugs, protein biosynthesis would be stopped with attenuation of bacterial growth. ${ }^{1}$ Currently, the aaRSs are interesting antibacterial drug targets, as remarkable increase in the prevalence of antibiotic resistance among bacteria. The aaRSs catalyze the attachment of specific amino acids to their cognate tRNAs, which is the initial reaction in protein biosynthesis. ${ }^{2}$ The aminoacylation reaction of tRNA proceeds in two steps: first, aaRSs activate their substrate amino acids by forming aminoacyl-adenylate. Second, the aminoacyl moiety from the adenylate is transferred to the 3 '-terminal adenosine of tRNA. ${ }^{3}$

$$
\mathrm{AA}+\mathrm{ATP}+\mathrm{tRNA} \rightarrow \mathrm{AA}-\mathrm{tRNA}+\mathrm{AMP}+\mathrm{PP}_{\mathrm{i}}
$$

Despite their conserved mechanisms, aaRSs are divided into two unrelated classes (I and II) each with ten enzymes. The respective classes have different sequence motifs and distinct ac-

\footnotetext{
${ }^{\top}$ This paper is dedicated to Professor Sunggak Kim on the occasion of his honorable retirement.

${ }^{a}$ Current address: Division of Structural and Computational Biology, School of Biological Sciences, Nanyang Technological University, 637551, Singapore. ${ }^{b}$ Current address: Biomolecular Modeling and Design Division, Bioinformatics Institute, 30 Biopolis Street, \#07-01 Matrix, 138671, Singapore.
}

tive site topologies. ${ }^{4,6}$ The active site of 10 Class I aaRSs is in the ATP-binding Rossman fold, whereas the site is constructed around an antiparallel $\beta$-sheet in class II aaRS enzymes. ${ }^{7}$ The accuracy of protein biosynthesis is determined by the correct recognition of specific amino acids and tRNAs by aaRS enzymes. ${ }^{8}$ To maintain high translational accuracy, these aaRS enzymes catalyze editing reactions by hydrolysing the incorrect products.

The editing reaction has been a great interest for study and these critically important proteins can be a strong contender for drug target for the antimicrobial agent development. ${ }^{9-14}$ In order to assess the binding affinity of inhibitor candidate molecules with aaRSs, molecular docking study was introduced. Four different scaffolds (scaffold 1-4) were generated by critical analyses of several crystal structures of aaRS amino-acylation binding pockets with substrates/ligands. Preliminary virtual library was generated comprising of 48 compounds as a sample to analyze the binding mode of the new hypothetical compounds. These compounds were developed based on the structural backbone of commercial antibiotic, mupirocin and the isoleucyl-adenylate reaction intermediate with isoleucyl moiety in the tail part. ${ }^{15,16}$ To investigate binding mode of aaRS with the developed derivatives, molecular docking simulations of four different scaffolds were carried out within the aminoacylation sites of eight different aaRSs (seven from E. coli and one from S. aureus).

\section{Methods}

Preparation for protein and ligand structures. The three-di- 
Table 1. Structural and basic information for the eight AARS proteins

\begin{tabular}{lcll}
\hline Target & Organism & Class & PDB ID \\
\hline IleRS & S. aureus & Class I & 1QU3 \\
MetRS & E. coli & Class I & 1PFY \\
GlnRS & E. coli & Class I & 1EUQ \\
TyrRS & E. coli & Class I & 1VBM \\
HisRS & E. coli & Class II & $1 \mathrm{KMM}$ \\
ThrRS & E. coli & Class II & $1 \mathrm{EVL}$ \\
AspRS & E. coli & Class II & 1C0A \\
LysRS & E. coli & Class II & 1EIT
\end{tabular}

mensional structures of the eight aaRSs, seven from E. coli and one from $S$. aureus, were obtained from Protein Data Bank (PDB): 1PFY for E. coli MetRS, 1C0A for E. coli AspRS, 1EVL for $E$. coli ThrRS, 1KMM for $E$. coli HisRS, 1EUQ for $E$. coli GlnRS, 1VBM for E. coli TyrRS, 1EIT for E. coli LysRS and 1QU3 for S.aureus IleRS, respectively (Table 1). ${ }^{17-24}$ Virtual library of 48 compounds used for this study were developed based on four scaffolds (Table 2-5) and followed by database generation carried out using MDL ISIS/Base and ISIS/Draw. ${ }^{25}$ All the 48 compounds are converted into 3D structures and performed energy minimization in Inisght II software before proceeding to molecular docking simulations.

Molecular docking simulation. Bio-molecular interactions and binding modes were analyzed using GOLD program. The GOLD 3.0.1 (Genetic Optimisation for Ligand Docking) from Cambridge Crystallographic Data Centre, UK, employs a genetic algorithm for docking of complete flexible ligands into partially flexible protein binding sites. ${ }^{26}$ Active site radiuses were taken as $10.0 \AA$ around center of the site within each aaRS protein. All four scaffold compounds were docked into active sites of each protein. The RMSD, annealing parameter values of van der Waals (VDW) and hydrogen bond interactions were considered within $1.5,4.0$, and $2.5 \AA$, respectively. ${ }^{27,28}$ The best docked conformations were selected based on the GoldScore ranking.

Analysis of docked conformations. The protein structure analyses and manipulations were performed using InsightII program. ${ }^{29}$ The GOLD results were analyzed by Silver 1.0 software which is a browser for visualizing protein-ligand docked conformation obtained. In order to understand interaction pattern (hydrophobic and hydrogen bonding interactions) between docked ligand and protein active site, Ligplot software was used as an essential tool. ${ }^{30}$

\section{Results and Discussions}

The main goal of this study was to design new inhibitors and thus to investigate the binding modes of the candidate molecules at the active site of aaRSs. Four different scaffolds for molecular docking simulation were designed. All of the compounds bear imidazolidin-2-one as a core heterocycle with substituent of amino acids at $\mathrm{N} 1$ position and urea or sulfonamide at $\mathrm{C} 4$ position as novel peptide mimics (Table 2-5). In case of scaffold 1 , comprising amino acids as substituent at N1 position along with acetic acid and urea at $\mathrm{C} 4$ position (Table 2). In scaffold 2, only
Table 2. Chemical structures for scaffold 1<smiles>[Y]C(C[C@H]1CN(C([R1])C(=O)O)C(=O)N1)NN</smiles>

\begin{tabular}{cccc}
\hline Compound & $\mathrm{R} 1$ & $\mathrm{R} 2$ & $\mathrm{X}$ \\
\hline 1 & $\mathrm{Me}$ & Ala & $\mathrm{O}$ \\
2 & $\mathrm{Me}$ & Leu & $\mathrm{O}$ \\
3 & $\mathrm{Me}$ & $\mathrm{Ile}$ & $\mathrm{O}$ \\
4 & $\mathrm{Me}$ & Val & $\mathrm{O}$ \\
5 & Phenyl & Ala & $\mathrm{O}$ \\
6 & Phenyl & Leu & $\mathrm{O}$ \\
7 & Phenyl & Ile & $\mathrm{O}$ \\
8 & Phenyl & Val & $\mathrm{O}$ \\
9 & Pyrimidine & Ala & $\mathrm{O}$ \\
10 & Pyrimidine & Leu & $\mathrm{O}$ \\
11 & Pyrimidine & Ile & $\mathrm{O}$ \\
12 & Pyrimidine & Val & $\mathrm{O}$ \\
\hline
\end{tabular}

Scaffold 1 (compound 1-12) * is R/S taken

Table 3. Chemical structures for scaffold 2<smiles></smiles>

\begin{tabular}{cccc}
\hline Compound & $\mathrm{R} 1$ & $\mathrm{R} 2$ & $\mathrm{X}$ \\
\hline 13 & $\mathrm{Me}$ & Ala & $\mathrm{O}$ \\
14 & $\mathrm{Me}$ & Leu & $\mathrm{O}$ \\
15 & $\mathrm{Me}$ & $\mathrm{Ile}$ & $\mathrm{O}$ \\
16 & $\mathrm{Me}$ & $\mathrm{Val}$ & $\mathrm{O}$ \\
17 & Phenyl & Ala & $\mathrm{O}$ \\
18 & Phenyl & Leu & $\mathrm{O}$ \\
19 & Phenyl & Ile & $\mathrm{O}$ \\
20 & Phenyl & Val & $\mathrm{O}$ \\
21 & Pyrimidine & Ala & $\mathrm{O}$ \\
22 & Pyrimidine & Leu & $\mathrm{O}$ \\
23 & Pyrimidine & Ile & $\mathrm{O}$ \\
24 & Pyrimidine & Val & $\mathrm{O}$
\end{tabular}

Scaffold 2 (compound 13-14) * is R/S taken

one moiety was changed from urea to sulfonamide at $\mathrm{C} 4$ in scaffold 1 (Table 3). Compounds in scaffold 3 include substituent of dipeptide at N1 and urea at C4 (Table 4). Scaffold 4 is same as scaffold 3 with an exception of urea moiety changed to sulfonamide at $\mathrm{C} 4$ position (Table 5). Alphatic side chain containing amino aicds like alanine, valine, leucine and isoleucines are incorporated at N1 position as a substitution. Forty eight com- 
Table 4. Chemical structures for scaffold 3

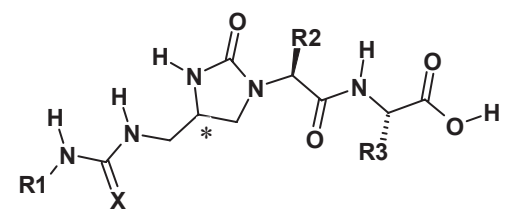

\begin{tabular}{ccccc}
\hline Compound & R1 & R2 & R3 & X \\
\hline 25 & Me & Ala & Ala & O \\
26 & Me & Leu & Leu & O \\
27 & Me & Ile & Ile & O \\
28 & Me & Val & Val & O \\
29 & Phenyl & Ala & Ala & O \\
30 & Phenyl & Leu & Leu & O \\
31 & Phenyl & Ile & Ile & O \\
32 & Phenyl & Val & Val & O \\
33 & Pyrimidine & Ala & Ala & O \\
34 & Pyrimidine & Leu & Leu & O \\
35 & Pyrimidine & Ile & Ile & O \\
36 & Pyrimidine & Val & Val & O \\
\hline
\end{tabular}

Scaffold 3 (compound 25-36) * is R/S taken

Table 5. Chemical structures for scaffold 4<smiles>[R3]C(C(=O)N[C@@H]([R3])C(=O)O[2H])N1C[C@H](CNS([R1])(=O)=O)N([2H])C1=O</smiles>

\begin{tabular}{ccccc}
\hline Compound & R1 & R2 & R3 & X \\
\hline 37 & Me & Ala & Ala & O \\
38 & Me & Leu & Leu & O \\
39 & Me & Ile & Ile & O \\
40 & Me & Val & Val & O \\
41 & Phenyl & Ala & Ala & O \\
42 & Phenyl & Leu & Leu & O \\
43 & Phenyl & Ile & Ile & O \\
44 & Phenyl & Val & Val & O \\
45 & Pyrimidine & Ala & Ala & O \\
46 & Pyrimidine & Leu & Leu & O \\
47 & Pyrimidine & Ile & Ile & O \\
48 & Pyrimidine & Val & Val & O \\
\hline
\end{tabular}

Scaffold 4 (compound $37-48$ ) * is R/S taken

pounds based on four different scaffolds were used to perform molecular docking simulations into the active sites of eight different aaRSs.

Comparison of interaction properties among four scaffolds. GOLD Molecular docking produces 10 probable binding conformations for each compound i.e., around 480 binding poses for each target (aaRS). All these conformations are evaluated care-

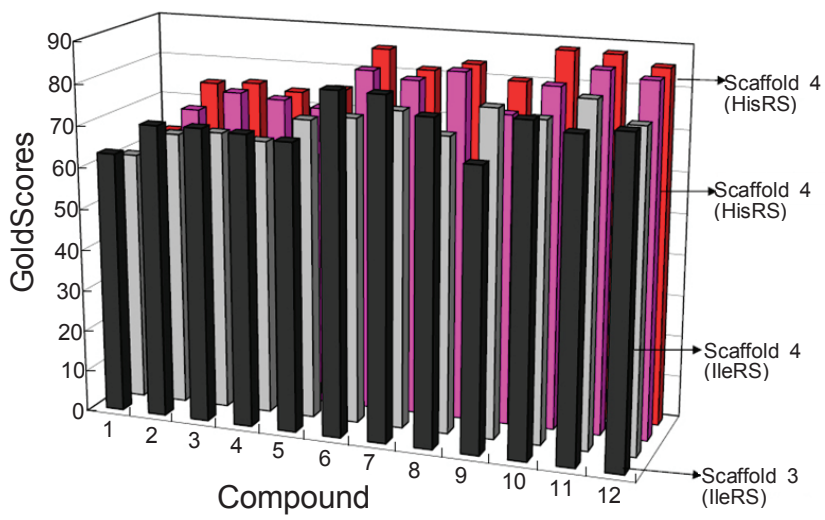

Figure 1. Comparison of GoldScores for scaffolds 3 and 4 with S. aureus IleRS and E. coli HisRS respectively. Compounds were renumbered as 1-12 for scaffold 3 (25-36) and scaffold 4 (37-48) for representing a comparison between them.

fully and selected based on two methods 1) binding pose similarity and interaction mode in reference with crystal ligand pose 2) Goldscore for the particular binding pose. This evaluation was carried out for all the eight protein targets. From all the eight targets, docking simulation results revealed that scaffolds 3 and 4 compounds demonstrated better GoldScores compared to scaffolds 1 and 2 compounds. The compounds (1-24) from scaffolds 1 and 2 were not able to cover full active site volume as these two scaffolds are having a single amino acid substitution at N1 position. Due to this reason these compounds are unable to form proper interactions with active site residues. Therefore these two scaffolds may not be appropriate inhibitors for the eight aaRS proteins.

The scaffold 4 compounds delivered comparatively better or equal dock scores to scaffold 3 (Figure 1). Among 12 compounds of scaffolds 3 and 4 , compounds containing methyl group at R1 position showed less score as well as poor interaction for the eight aaRS proteins. Phenyl and pyrimidine group at R1 position enhanced the dock score. Except for alanine side chain, others (Val, Leu, Ile) at R2 and R3 positions showed better dock scores. Therefore phenyl/pyrimidine groups at R1 position and bulky side chains at $\mathrm{R} 2$ and $\mathrm{R} 3$ positions are favorable for both scaffolds 3 and 4 .

Based on the overall interactions of all conformations for all the scaffolds, scaffolds 3 and 4 are having good interactions with seven E. coli aaRS and one S. aureus IleRS. Among them compounds with phenyl/pyrimidine at R1 position and bulky groups at R2 and R3 are having favorable interactions. These compounds had consistently better binding properties, specifically for HisRS (E. coli) and IleRS (S. aureus).

Molecular docking results of HisRS (E. coli). Analyses of Histidyl-adenylate (His-AMS) interaction with HisRS crystal structure (PDB ID : 1KMM) were carried out to understand original binding mode between substrate and HisRS. The binding mode was formed by 13 hydrogen bonds and many hydrophobic interactions. Hydrogen bond interactions with R 259 were observed, which is an important conserved amino acid in all HisRSs. Histidine ring was found to interact with Y264, E131, and T85, respectively (Figure 2 ). 


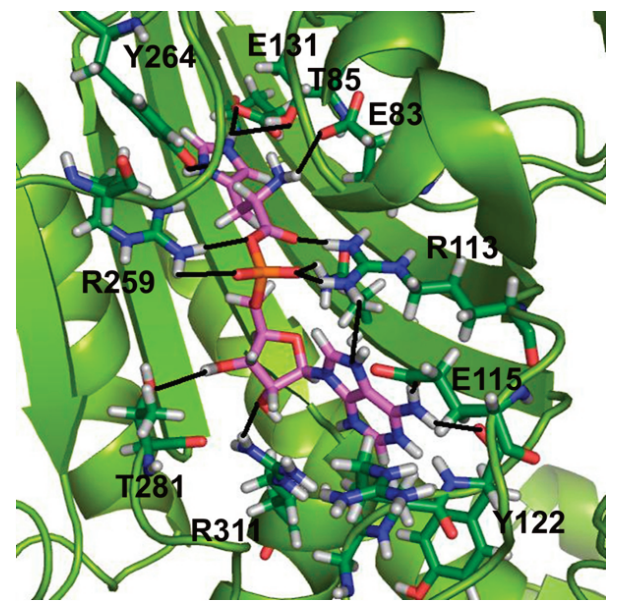

Figure 2. Crucial interactions between His-AMS crystal ligand (pink) and HisRS target protein (green). The HisRS residues interacting with the His-AMS are shown in stick model. Hydrogen bonding interactions were represented as line.
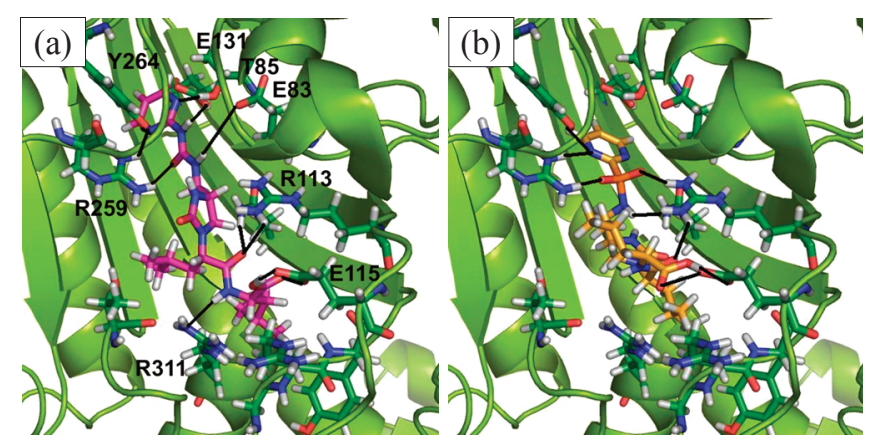

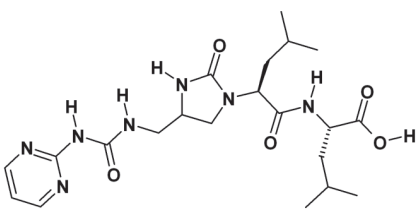

Compound 34 (scaffold 3)

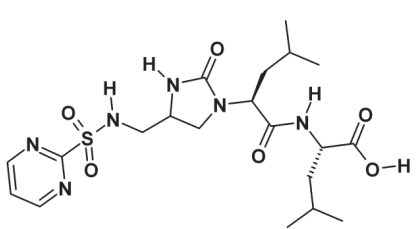

Compound 46 (scaffold 4)
Figure 3. Binding modes between compound 34 (a), compound 46 (b) and HisRS target protein. Similar type of interactions observed like His-AMS (shown as Figure 2).

Molecular docking results for compound 34 and 46 which are the best GoldScore compounds for scaffolds 3 and 4 were analyzed with HisRS active site residues. Hydrogen bonding interactions were noticed with R259. Pyrimidine ring of compound 34 and 46 interacted with Y264, E131, and T85. Interactions formed with other important amino acids were similar to that of experimentally proven crystal ligand (Figure 3 ). Hence, the overall binding modes of compound 34 and 46 were similar with that of crystal ligand form.

Molecular docking results of IleRS (S. aureus). Analyses of mupirocin interaction with IleRS crystal structure (PDB ID: 1QU3) were performed to identify original binding mode between existing inhibitor and IleRS. Heterocylic ring or 3,4-di hydroxy tetrahydro-pyran or monate core of the mupirocin were observed to interact tightly by hydrogen bonding with the following residues: Gly554, Glu555, Asp557, and Gln558. Carbonyl group of mupirocin interacts with Val588 through hydro-

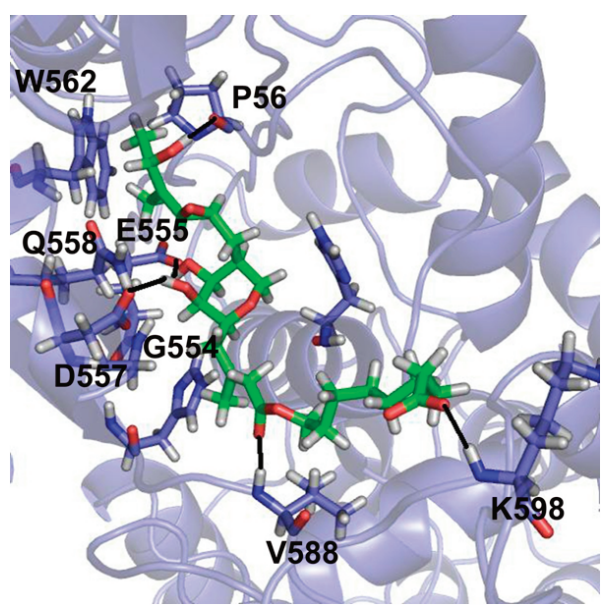

Figure 4. Crucial interactions between mupirocin crystal ligand (green) and IleRS target protein (light blue). The IleRS residues interacting with the mupirocin are shown in stick model. Hydrogen bonding interactions were represented as line.
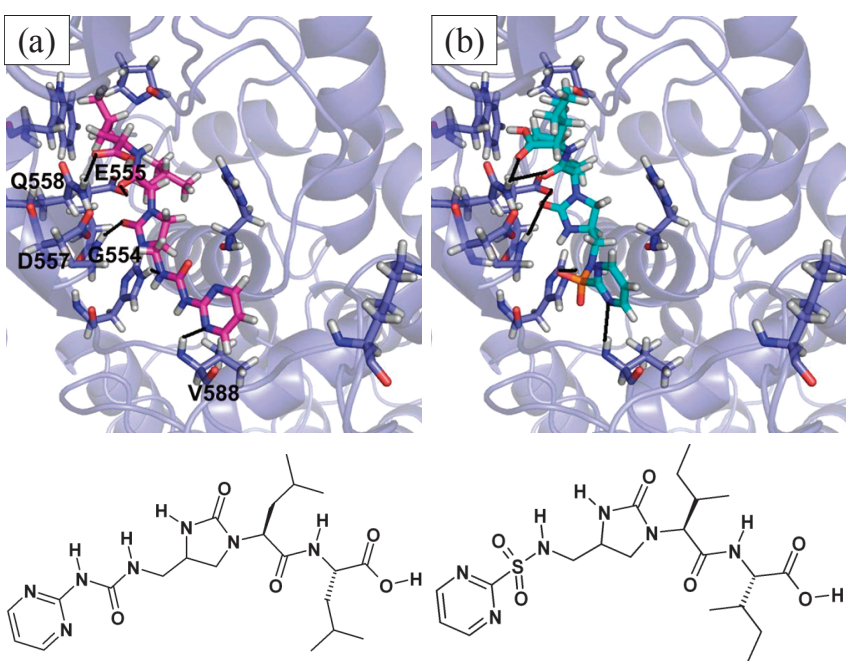

Compound 34 (scaffold 3)

Compound 47 (scaffold 4)

Figure 5. Binding modes between compound 34 (a), compound 47 (b) and IleRS target protein. Similar type of interactions observed like mupirocin (shown as Figure 4).

gen bonding interaction. Trp562 is a crucial amino acid for hydrophobic interactions. Terminal carboxylic group in mupirocin interacts with second lysine of KMSKS motif (Figure 4).

Molecular docking results for compound 34 and 47 which are the best GoldScore compounds for scaffolds 3 and 4 were employed with IleRS active site residues. These compounds interacted with Gly554, Glu555, Asp557, and Gln558 residues by hydrogen bonds. Pyrimidine ring of compounds 34 and 47 interacted with Val588. Amino acid side chains at R3 position were observed to interact with Trp562 through hydrophobic interactions (Figure 5).

Another interesting point was observed upon comparison of binding modes of scaffold 3 and 4 compounds with $E$. coli HisRS and $S$. aureus IleRS. In the case of HisRS, the pyrimidine ring of the compounds occupies the immidazole ring position of substrate and forms hydrophobic and hydrogen bond interactions with the HisRS. Surprisingly in the case of IleRS, py- 
rimidine ring occupies the adenosine binding pocket and substitutes the purine ring and interacts with Val588. Other side of the pocket which can accommodate only aliphatic side chain (Ile) is substituted with R3 groups (Ile or Leu side chains) in a similar manner like mupirocin or Ile-AMS. This analysis demonstrates that these compounds can interact with several aaRSs and can be used as inhibitors of different aaRSs.

\section{Conclusions}

Four different scaffolds (scaffold 1-4) were generated by analyzing the available crystal structures of aaRS amino-acylation binding pockets with substrates/ligands. Virtual library of 48 compounds used for this study were developed based on four scaffolds (Table 2-5) and developed a database for further use. To investigate binding ability of these 48 hypothetical derivatives, molecular docking simulations were carried out within the active/aminoacylation sites of eight different aaRSs (seven from $E$. coli and one from $S$. aureus). Compounds from the first two scaffolds may not be appropriate inhibitors for the eight aaRS proteins as these compounds possess only single amino acid substitution at N1 position and are unable to cover the active site volume. Scaffolds 3 and 4 are having good interactions with seven $E$. coli aaRS and one $S$. aureus IleRS. Among them compounds with phenyl/pyrimidine at R1 position and bulky groups at R2 and R3 are having favorable interactions. These compounds have consistently better binding properties, specifically for HisRS (E. coli) and IleRS (S. aureus). Based on GOLD docking score comparison, it can be concluded that they may have better affinity towards $E$. coli HisRS than $S$. aureus IleRS. The binding modes of the best compounds within two proteins were well compatible with that of each crystal ligand. Finally, we suggest that final compounds have good inhibitory activity for in vitro aaRS. These observations are beneficial to design new antibacterial lead candidates.

Acknowledgments. Students were recipients of fellowships from the BK21 Programs. This work was also supported by a grant from the MEST/NRF to the Environmental Biotechnology National Core Research Center (20090091489) and Pioneer Research Center Program (2009-0081539) and by financial support from GRRC program (2009B01). HJH acknowledges the financial support from GRRC program of Gyeonggi province (2009B01).

\section{References}

1. Ruan, B.; Bovee, M. L.; Sacher, M.; Stathopoulos, C.; Poralla, K.;
Francklyn, C. S.; Söll, D. J. Biol. Chem. 2005, 280, 571.

2. Fersht, A. R.; Kaethner, M. M. Biochemistry 1976, 15, 3342.

3. Forrest, A. K.; Jarvest, R. L.; Mensah, L. M.; O'Hanlon, P. J.; Pope, A. J.; Sheppard, R. J. Bioorg. Med. Chem. Lett. 2000, 10, 1871.

4. Eriani, G.; Delarue, M.; Poch, O.; Gangloff, J.; Moras, D. Nature 1990, 347, 203.

5. Burbaum, J. J.; Schimmel, P. J. Biol. Chem. 1991, 266, 16965.

6. Cusack, S.; Härtlein, M.; Leberman, R. Nucleic Acids Res. 1991, 19, 3489.

7. Arnez, J. G.; Moras, D. Trends. Biochem. Sci. 1997, 22, 211.

8. Shen, N.; Guo, L.; Yang, B.; Jin, Y.; Ding, J. Nucleic Acids Res. 2006, 34, 3246.

9. Schmidt, E.; Schimmel, P. Science 1994, 264, 265.

10. Lin, L.; Hale, S. P.; Schimmel, P. Nature 1996, 384, 33.

11. Lin, L.; Schimmel, P. Biochemistry 1996, 35, 5596.

12. Fukunaga, R.; Fukai, S.; Ishitani, R.; Nureki, O.; Yokoyama, S. J. Biol. Chem. 2004, 279, 8396.

13. Fukunaga, R.; Yokoyama, S. J. Mol. Biol. 2005, 346, 57.

14. Lee, K. W.; Kwon, Y. J.; Briggs, J. M. Bull. Korean Chem. Soc. 2007, 28, 207.

15. Hurdle, J. G.; O’Neill, A. J.; Ingham, E.; Fishwick, C.; Chopra. I. Antimicrob. Agents Chemother. 2004, 48, 4366.

16. Nakama, T.; Nureki, O.; Yokoyama, S. J. Biol. Chem. 2001, 276, 47387.

17. Crepin, T.; Schmitt, E.; Mechulam, Y.; Sampson, P. B.; Vaughan, M. D.; Honek, J. F.; Blanquet, S. J. Mol. Biol. 2003, 332, 59.

18. Eiler, S.; Dock-Bregeon, A.; Moulinier, L.; Thierry, J. C.; Moras, D. EMBO. J. 1999, 18, 6532.

19. Sankaranarayanan, R.; Dock-Bregeon, A. C.; Rees, B.; Bovee, M.; Caillet, J.; Romby, P.; Francklyn, C. S.; Moras, D. Nat. Struct. Biol. 2000, 7, 461.

20. Arnez, J. G.; Augustine, J. G.; Moras, D.; Francklyn, C. S. Proc. Natl. Acad. Sci. US A 1997, 94, 7144.

21. Sherlin, L. D.; Bullock, T. L.; Newberry, K. J.; Lipman, R. S.; Hou, Y. M.; Beijer, B.; Sproat, B. S.; Perona, J. J. J. Mol. Biol. 2000, 299, 431.

22. Kobayashi, T.; Takimura, T.; Sekine, R.; Kelly, V. P.; Kamata, K.; Sakamoto, K.; Nishimura. S.; Yokoyama. S. J. Mol. Biol. 2005, $354,739$.

23. Omecinsky, D. O.; Holub, K. E.; Adams, M. E.; Reily, M. D. Biochemistry 1996, 35, 2836.

24. Silvian, L. F.; Wang, J.; Steitz, T. A. Science 1999, 285, 1074.

25. The MDL ISIS/base is available from MDL Information Systems Inc.: San Leandro, CA, http://www.mdl.com

26. Jones, G.; Willett, P.; Glen, R. C.; Leach, A. R.; Taylor, R. J. Mol. Biol. 1997, 267, 727.

27. Bharatham, N.; Bharatham, K.; Lee, K. W. Bull. Korean Chem. Soc. 2007, 28, 200.

28. Bharatham, N.; Bharatham, K.; Lee, K. W. Bull. Korean Chem. Soc. 2006, 27, 266.

29. InsightII, Version 2005.3L, Accelrys Inc., San Diego (www. accelrys.com)

30. Wallace, A. C.; Laskowski, R. A.; Thornton, J. M. Protein. Eng. $1995,8,127$. 